\title{
Crítica a la estructura tributaria actual en México
}

\author{
Eduardo Ramírez Cedillo*
}

\section{Resumen}

El presente documento tiene por objeto realizar una revisión crítica de la política tributaria en México, partiendo de dos puntos principales: las características de los principales impuestos y las características de una estructura tributaria eficiente. Tal como está diseñada la estructura tributaria del país resulta clara su ineficiencia para cerrar la brecha entre los ingresos potenciales y los reales, además de su incapacidad para lograr la distribución del ingreso. Ante tal situación se ha intentado el cambio de los tributos sobre la renta por los dedicados al consumo y su generalización, tratando de evitar con ello la evasión fiscal. A pesar de que se concede que puede resultar un sistema tributario regresivo se asume que puede ser subsanado mediante una correcta aplicación del gasto.

Palabras clave: Impuestos, Estructura Tributaria, Eficiencia Tributaria, Distribución del Ingreso, Equidad Tributaria

Fecha de recepción: 11/01/2006

Fecha de aceptación:11/01/2007

\section{Criticism of the Current Tax Structure in Mexico}

\begin{abstract}
The objective of this paper is to make a critical review of the tax policy in Mexico, considering of two main issues: the characteristics of the main taxes and the characteristics of an efficient tax structure. As it is designed, the tax structure of the country is clearly inefficient in order to reduce close the gap between potential income and the real one. In addition to its incapacity to obtain the distribution of the income. Under these circumstances it has been tried to exchange the tributes on the rent for those targeting consumption and their generalization. This in way fiscal evasion is
\end{abstract}

\footnotetext{
* Profesor de la Facultad de Contaduría y Administración y de la Universidad Tecnológica de México. Correo electrónico: ramceed@prodigy.net.com, ramceed@hotmail.com
} 
prevented. Although one grants that it can be a regressive tax system it is assumed that it can be corrected by means of the right application of the cost.

Keywords: Taxes, Tributary Structure, Tributary Efficiency, entrance distribution, Tributary Equity.

\section{Introducción}

Si se parte de la idea de que cualquier impuesto disminuye el bienestar de la sociedad o impone costos adicionales a sus actividades, dependiendo de cual sea su base gravable, se puede concluir que los impuestos son malos; no obstante si se piensa desde una óptica diferente, asumiendo que los recursos que el gobierno retira de la economía con su mano izquierda, son devueltos con su mano derecha vía bienes y servicios (que por sus características no son objeto del interés de los agentes privados o cuando si lo son su asignación tiende a ser sub-óptima), se estaría dándo sentido a la función tributaria del gobierno pensando que lo que hace es coordinar de manera estratégica la canalización de recursos con fines sociales y económicos para lograr: mejores niveles de vida, crecimiento económico y una redistribución del ingreso y la riqueza en una proporción “justa”. ${ }^{1}$ Siguiendo el argumento, se podría asegurar, como lo hacen algunos autores, que los efectos negativos de los impuestos en la sociedad pueden ser compensados por los efectos positivos del gasto del gobierno (Gemmell, 2001; Zangler y Dürnecker, 2003).

Sin embargo, cuando se cuenta con una estructura tributaria ineficiente o no óptima se pueden tener al menos dos problemas; el primero consiste en la diferencia entre los recursos que se extraen de la sociedad y la cantidad que pueden ser regresados, mientras que el segundo puede referirse a la combinación de gravámenes con los que se constituye la estructura tributaria.

En el primer caso se incrementa el gravamen al que son sometidos los contribuyentes, es decir, por cada peso que se extrae de la sociedad se regresa una proporción menor en virtud de los costos directos o indirectos en los que se incurren. Mientras que en el segundo, una combinación de impuestos que distorsione en mayor medida la economía puede tener serias consecuencias para asegurar su crecimiento a futuro y, por lo tanto, también pone en peligro la recaudación futura. Adicionalmente, una mala combinación de gravámenes puede tener un efecto

\footnotetext{
${ }^{1}$ Musgrave (1995: 7-15) menciona que la política fiscal debería de cumplir tres funciones: de Asignación, de Distribución y de Estabilización. Donde la segunda de ellas corresponde a la necesidad de ajustar el ingreso y la riqueza a lo que la sociedad considera equitativo y justo.
} 
Crítica a la estructura tributaria actual en México

serio en la redistribución del ingreso y la riqueza, dependiendo si se logra una estructura tributaria progresiva o regresiva.

En ambos casos la estructura tributaria deja de cumplir con su objetivo, tanto en su función de obtención de ingresos para sufragar los gastos del gobierno como en su función de redistribución del ingreso y la riqueza. La estructura fiscal de México no cumple con su cometido ya que es insuficiente para sufragar los gastos del gobierno, está lejos de su recaudación potencial y, en términos absolutos, resulta inequitativa.

En la teoría fiscal se abordan de manera interesante dos temas: ¿Cuáles son los requisitos que debe observar una estructura fiscal eficiente? y, ¿qué tipos de impuestos son los más idóneos o los menos perjudiciales para el crecimiento económico?² En este ensayo se hará una revisión de estos elementos con una visión crítica para el ámbito doméstico. En este sentido el documento se compone de la siguiente manera: Un punto II llamado Antecedentes de los principales impuestos en México, capacidades de recaudación, características y problemas, en él se darán algunas cifras de la recaudación del país; Punto III Estructura tributaria, donde se detallarán los elementos que deberá contener una estructura tributaria eficiente; Punto IV La propuesta de reforma realizada por el Ejecutivo Federal y otras alternativas, y por último, un punto $\mathrm{V}$ dedicado a las conclusiones.

\section{Antecedentes de los principales impuestos en México, capacidades de recaudación, características y problemas}

Los ingresos tributarios del país para el periodo de 1977-2003 representaron en promedio el 10.6\% del PIB con una variación que fue del $8.9 \%$ al $11.6 \%$ del PIB en sus valores mínimos y máximos respectivamente (gráfica 1). Para este periodo el impuesto sobre la renta (ISR) representó en promedio cerca del 44\% del total de los tributos, mientras que el Impuesto al Valor Agregado (IVA) integraba en promedio alrededor del 28\% y el Impuesto Especial sobre Producción y Servicios (IEPS) participaba en promedio con el 17\%. Alrededor del $89 \%$ del total de los ingresos tributarios en promedio se encontraba agregado en estos tres impuestos. ${ }^{3}$

\footnotetext{
2 Bajo la teoría del crecimiento endógeno (Romer, 1990) se ha avanzado mucho en la consideración del conjunto de impuestos que permiten o impiden el crecimiento, al respecto se puede revisar a los siguientes autores Easterly y Rebelo (1993), Engen y Skinner (1996), Myles (2000), Zangler y Durnecker (2003), entre otros.

${ }^{3}$ A partir del 2001 los tres impuestos mencionados representan más del 92\% del total de los ingresos tributarios y llevan una tendencia ligera pero ascendente (92.3, 92.4 y $92.5 \%$ para los años de 2001, 2002 y 2003 respectivamente).
} 
Gráfica 1

Ingresos tributarios como proporción del PIB

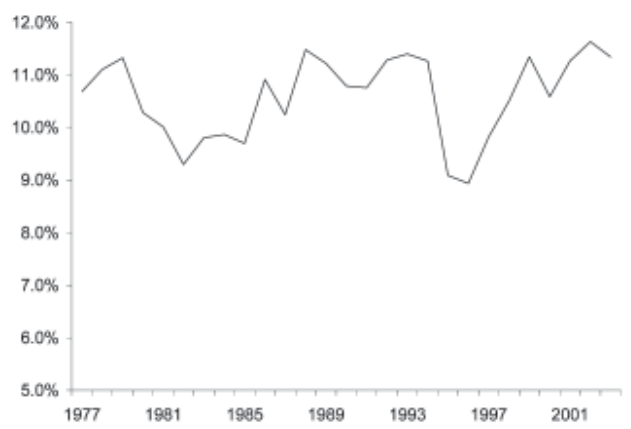

Fuente: Elaboración propia con datos del Sistema de Finanzas y Deuda Pública, Subsecretaría de Hacienda y Crédito Público y Dirección General de Planeación Hacendaria.

Las variaciones observadas por los ingresos tributarios obedecen al comportamiento del ciclo económico. Como se menciona en la teoría tributaria, los ingresos tributarios son de carácter pro-cíclico, es decir, que cuando la economía crece los ingresos tributarios también lo hacen. Hernández et al. (2000) menciona que hay una relación directa entre el crecimiento económico y la recolección de impuestos, al comentar la disminución de los mismos derivada de la crisis de 1995. En la gráfica 2 se puede apreciar la relación del crecimiento económico y los ingresos tributarios; la correlación que guardan ambas variables es cercana al 0.96 para el periodo de referencia. Un aspecto interesante de comentar es que precisamente en el año que se registran los mínimos históricos de recaudación son precisamente en 1995 y 1996 (gráficas 1 y 2).

Gráfica 2

Relación del comportamiento de los ingresos tributarios y el PIB en términos constantes a precios de 1993

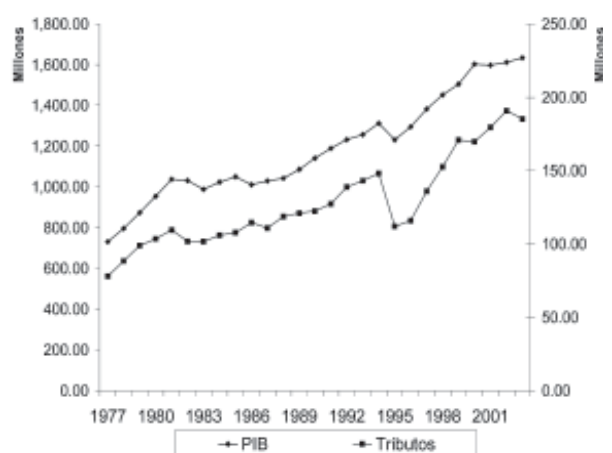

Fuente: Elaboración propia con datos del Sistema de Finanzas y Deuda Pública, Subsecretaría de Hacienda y Crédito Público, Dirección General de Planeación Hacendaria e INEGI. 
Crítica a la estructura tributaria actual en México

Analizando los tres impuestos antes mencionados, su respuesta al nivel de producto agregado es diferente. Por una parte cuando se analiza la correlación de las series en logaritmos se aprecia que el IVA tiene una amplia correlación con el producto nacional aproximadamente en 95\%, mientras que el ISR se correlaciona en $88 \%$ y el IEPS incluso presenta una correlación de $64 \%$. Si se analiza la correlación con base en las primeras diferencias de los datos (tasas de crecimiento), se aprecia que los resultados cambian y que en este caso es el ISR el que tiene una correlación más importante con el producto nacional en aproximadamente el 67\%, mientras que la correlación del IVA disminuye al 32\% y la correlación del IEPs se presenta con signo negativo -16\% (cuadro 1).

Los resultados de las correlaciones son una evidencia del comportamiento de los impuestos seleccionados con relación al producto agregado, con lo cual se tiene que el ISR resulta más procíclico que los otros impuestos dedicados al consumo y por ello si se quiere mantener el ingreso tributario independientemente de las variaciones del producto. Una medida a seguir consistiría en el cambio de impuestos sobre la renta por impuestos al consumo, situación que es consistente con los planteamientos de organismos internacionales y con la propuesta del gobierno encabezado por el presidente Fox. A continuación se hace una revisión de los tres impuestos en cuestión.

Cuadro 1

Correlación del PIB los impuestos más representativos

\begin{tabular}{|c|c|c|c|c|}
\hline \multicolumn{5}{|c|}{ Matriz de correlación en logaritmos } \\
\hline & PIB & IEPS & IVA & ISR \\
\hline PIB & 1.00 & & & \\
\hline IEPS & 0.64 & 1.00 & & \\
\hline IVA & 0.95 & 0.71 & 1.00 & \\
\hline ISR & 0.89 & 0.51 & 0.80 & 1.00 \\
\hline \multicolumn{5}{|c|}{ Matriz de correlación primeras diferencias } \\
\hline & $\begin{array}{l}\text { PIB } \\
1.00\end{array}$ & IEPS & IVA & ISR \\
\hline IEPS & -0.16 & 1.00 & & \\
\hline IVA & 0.32 & -0.29 & 1.00 & \\
\hline ISR & 0.67 & 0.05 & -0.01 & 1.00 \\
\hline
\end{tabular}

Fuente: Elaboración propia con datos del Sistema de Finanzas y Deuda Pública, Subsecretaría de Hacienda y Crédito Público y Dirección General de Planeación Hacendaria.

\section{Impuesto al Valor Agregado}

El IVA tuvo su antecedente más próximo en el Impuesto Federal Sobre Ingresos Mercantiles (ISIM), implantado en el año de 1948, cuyo propósito fue sustituir el impuesto general del timbre que resultaba obsoleto para gravar el comercio y la industria 
(Ayala 2001: 255). El ISIM contemplaba en su inicio una sola tasa del 3\% sobre el importe a la enajenación; posteriormente, en 1971, se le incorpora la tasa especial del 10\% aplicable a productos no necesarios; en 1973 se adopta la tasa del 4\% que sustituye a la del 3\%; en 1974 se incorporan las tasas del 5, 15 y 30\% para automóviles y, para 1978 se incluye la tasa del 7\% (Ayala, 2001: 262). ${ }^{4}$

Hernández et al. (2000) menciona que las modificaciones realizadas a la Ley del IVA han servido para mantener de manera estable la recaudación de ingresos obtenidos mediante este impuesto (véase gráfica 3). Sin embargo, un elemento importante a destacar es que la productividad del impuesto, en los términos expuestos por Trigueros y Fernández (2001), no ha mostrado una mejoría relevante.

Gráfica 3

Relación del comportamiento de los ingresos por concepto de IVA y el PIB en términos constantes a precios de 1993

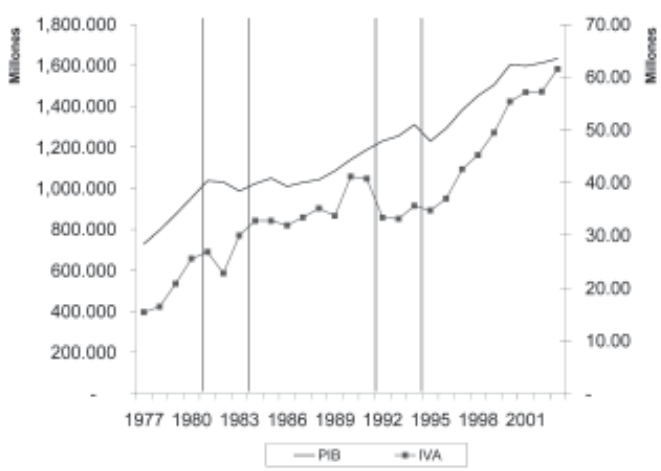

Fuente: Elaboración propia con datos del Sistema de Finanzas y Deuda Pública, Subsecretaría de Hacienda y Crédito Público y Dirección General de Planeación Hacendaria.

La productividad del IVA en promedio para el periodo 1977-2003 fue de 22.7\% (véase gráfica 4), es decir, de cada 100 pesos que potencialmente podrían ser recaudados tan sólo se recaudaron cerca de 23 pesos; la razón consiste en las diferentes tasas que se manejan y el régimen de exenciones, que por una parte disminuyen la base gravable y que por otra permiten la evasión del impuesto. ${ }^{5}$ Por lo tanto, una de las muchas propuestas para modificar y hacer más eficiente el IVA consiste en la unificación de las tasas y el abandono del régimen de exención. Hernández y Zamudio (2004) mencionan que los regímenes de exenciones y tratamientos especiales son comunes en el mundo, pero que a diferencia de México el resto de los países de la OCDE manejan

${ }^{4}$ Para un desarrollo de la evolución del sistema impositivo antes de la reforma véase Gil y Thirsk (2000)

${ }^{5}$ Bajo este argumento encontramos bastante similitud entre diversos autores, como son: Gil y Thirsk (2000), Trigueros y Fernández (2001), Hernández y Zamudio (2004), etcétera. 
tratos preferenciales con tasas positivas, por lo que la productividad de su impuesto no se ve tan mermada como la nuestra; el autor menciona el caso de Corea que presenta tratamientos similares al caso de México, pero su productividad es de 35\%.

Es importante destacar que el IVA en la década de los ochentas representaba el 27.8\% del total de los ingresos tributarios, mientras que en la década de los noventas en promedio participó con el 29\% del total y, ya para el periodo de 2000-2003, su participación fue del 31.9\%. ${ }^{6}$

Gráfica 4

Productividad del IVA

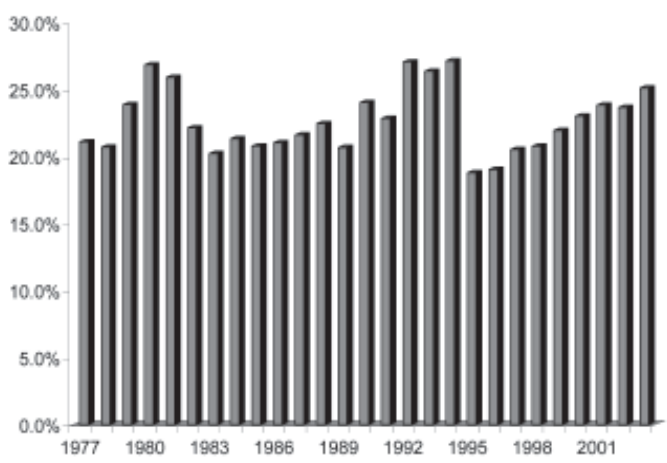

Fuente: Elaboración propia con datos del Sistema de Finanzas y Deuda Pública, Subsecretaría de Hacienda y Crédito Público y Dirección General de Planeación Hacendaria.

Nota: En los años de 1992-1994 se puede apreciar la mayor productividad de los últimos 15 años, sin embargo esto se debe a la disminución de la tasa del 15 al $10 \%$, un dato interesante es el incremento en la productividad a partir de 1996 a 2003.

Base del impuesto:

La base del IVA está definida como la suma del gasto en consumo de bienes y servicios de los residentes de una economía. ${ }^{7}$

\section{Ventajas:}

$>$ Grava el ingreso sólo cuando éste se consume.

$>$ No desalienta la acumulación de capital.

$>$ Tiene gran potencial de recaudación.

${ }^{6}$ Ibarra (2001) argumenta al respecto que este incremento en la participación del IVA en la recaudación total obedece a un cambio en la economía mundial que se ve reflejado en una apertura de las fronteras, por lo tanto es necesario buscar ingresos adicionales que puedan cubrir esos espacios.

7 La Ley del Impuesto al Valor Agregado establece en su Artículo $1^{\circ}$ que están obligados al pago del impuesto al valor agregado, establecido en dicha Ley, las personas físicas y morales que en territorio nacional realicen los actos o acciones siguientes: I) Enajenen bienes, II) Presten servicios independientes, III) Otorguen el uso o goce temporal de bienes y IV) Importen Bienes y Servicios. 
> Es relativamente simple su administración por lo que implica bajos costos.

$>$ Tiene la capacidad de gravar actividades informales.

\section{Desventajas:}

$>$ Desalienta el esfuerzo laboral al disminuir el ingreso disponible.

$>$ Resulta difícil su aplicación general por lo que es necesario la introducción de exenciones.

$>$ Poco efectivo para propiciar la equidad vertical.

$>$ El uso de tratamientos especiales atenta contra sus ventajas ya que disminuye la base gravable, diminuye el potencial, se complica su administración incrementando su costo y puede facilitar la evasión.

Uno de los elementos que explican la evasión del IVA, de acuerdo con Hernández y Zamudio (2004), es la forma en que se audita, pues cuando la posibilidad de ser auditado es baja se producen incentivos para sub-reportar ingresos o sobre-reportar deducciones. Sin embargo, no hay que olvidar que se debe ser cauteloso en esta materia, ya que la única forma de comprobar el cabal cumplimiento de las obligaciones sería la realización de auditorias a todo el padrón de contribuyentes pero seguramente eso implicaría más costos que los que representa la actual evasión; por lo tanto, es importante jugar con la posibilidad de la auditoria y valorar la posibilidad de incrementar las sanciones. ${ }^{8}$

Impuesto Sobre la Renta (ISR)

Definitivamente el impuesto más complejo de la estructura tributaria y por el que se recaudan los mayores ingresos tributarios resulta ser el ISR; en ese contexto, para algunos autores la complejidad del impuesto está relacionada a la búsqueda de un impuesto que resulte progresivo. ${ }^{9}$ Díaz y Mendoza (2005) mencionan que en el caso del ISR de ingresos por salarios parecería que la complejidad se deriva de la intención de conciliar la obligación del pago de los impuestos con el cumplimiento social de otorgar exenciones a sectores de la población y a ciertos rubros de ingresos sobre la base de una estructura salarial excesivamente polarizada.

\footnotetext{
${ }^{8}$ Hernández y Zamudio (2004) agregan que la evasión fiscal también puede ocurrir por la percepción negativa de los contribuyentes al respecto del gasto que realiza el Sector Público y el hecho de que la carga fiscal no sea equitativa.

${ }^{9}$ Rosen (2002: 254-255) comenta que la definición de un impuesto progresivo no es tan sencilla y que su complejidad avanza cuando no se establece con base en qué se determina la progresividad del impuesto, si es en relación al tipo impositivo medio o con base en el tipo impositivo marginal. Además menciona que medir la progresividad de un sistema fiscal puede ser más complicado que su definición.
} 
Crítica a la estructura tributaria actual en México

Ayala (2001: 259-261) expone que el antecedente más remoto del ISR es la Ley del 20 de junio de 1921, que estableció el gravamen conocido como el impuesto del centenario; una de sus características es que fue de carácter transitorio, a diferencia del que se estableció en la Ley del 21 de febrero de 1924 que fue de carácter permanente y que se puede considerar como la disposición que define la implantación del impuesto. Posteriormente se tuvieron algunas adecuaciones de importancia en los años de 1953, 1964, 1980, 1991, etcétera.

Por concepto de ISR el país, en promedio durante 1977-2003, ha recaudado cerca del $44 \%$ (véase gráfica 5) del total de los ingresos tributarios: en los ochentas se recaudaba el 42.8\%; en la década de los noventas la participación del ISR se incrementó casi en un punto porcentual al situarse en promedio alrededor del 43.7\%, del 2000 al 2003 se registró en promedio un nuevo avance al situarse en $43.9 \%$ del total de la recaudación.

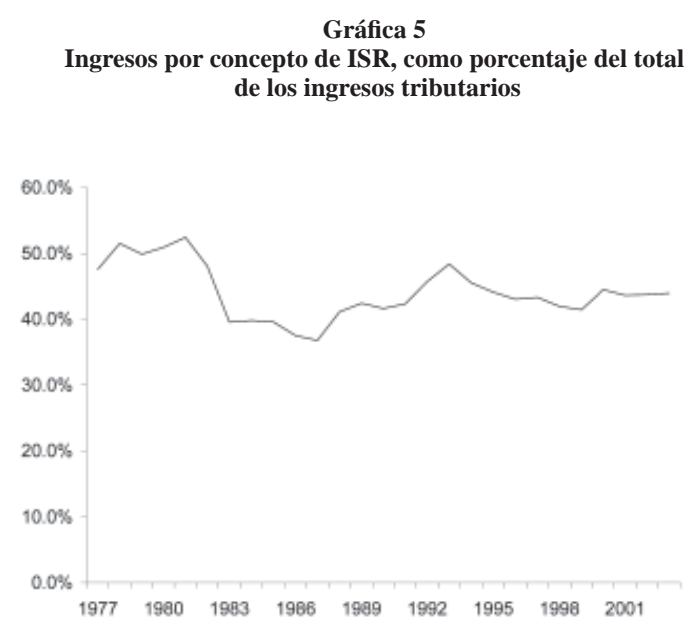

Fuente: Elaboración propia con datos del Sistema de Finanzas y Deuda Pública, Subsecretaría de Hacienda y Crédito Público y Dirección General de Planeación Hacendaria.

Dado el nivel de captación del ISR se constituye como uno de los impuestos más importantes para estructura tributaria, no obstante los efectos que puede tener sobre los agentes económicos, la variación de su recaudación atada a las fluctuaciones económicas y su alto margen de elusión y evasión fiscal son elementos que deben considerarse. 
Eduardo Ramírez Cedillo

Base del impuesto:

La base de este impuesto está definida como la suma de las remuneraciones al trabajo, las rentas y las utilidades generadas por los activos físicos y los intereses, los dividendos y las ganancias de capital devengados por los activos financieros.

Ventajas:

> Tiene una alta relación con la capacidad de pago de los contribuyentes.

$>$ Crea una base para introducir progresividad en el sistema tributario, a través de tasas que aumentan con el nivel de ingreso.

Desventajas:

> Dificultad para establecer la base, lo que origina la constante elusión fiscal.

$>$ La equidad vertical no es del todo aplicable a lo largo del ciclo de vida.

$>$ Tratamiento asimétrico a ganancias y pérdidas de las empresas.

$>$ Desalienta la acumulación de capital físico y humano.

$>$ Alienta las actividades informales.

Al igual que en el caso del IVA, el ISR es una coladera por donde se diluyen gran parte de los recursos que deberían ser ingresados al fisco federal; las razones tienden a ser similares: una cantidad considerable de tratamientos especiales y exenciones poco justificadas. Cantalá et al. (2005) menciona que en el caso del ISR dirigido a personas físicas, las exenciones y los tratamientos especiales no sólo reducen la recaudación potencial, sino que también complican y dificultan la actividad recaudatoria. El autor abunda en algunas medidas complementarias que pueden realizarse ya sea para incrementar la recaudación o para evitar la evasión:

- En el primer caso se podría incrementar la base tributaria ya que se tiene un gran universo de personas en el sector informal; sin embargo, ésta resulta ser una alternativa poco viable por la gran dimensión del problema y sus repercusiones sociales, además de que en algunos casos el sector informal por el nivel de ingresos que tiene no incrementaría de manera considerable los ingresos tributarios. Por lo tanto, quizás sea más conveniente enfocarse a la evasión del sector formal, argumenta Díaz y Mendoza (2005), quien menciona adicionalmente que en términos de equidad debe hacerse un esfuerzo para integrar a todos los trabajadores del sector informal al padrón de contribuyentes.

- En el segundo caso, al igual que se argumentaba para el IVA, la evasión puede ser disminuida si el contribuyente percibe que el ilícito tiene una alta probabilidad de ser detectado; adicionalmente se debe intentar el pago voluntario de los impuestos usando como medida de convencimiento la mejora en los servicios que proporciona el Gobierno. 
Crítica a la estructura tributaria actual en México

\section{Impuesto Especial sobre Producción y Servicios (IEPS)}

El IEPS propiamente se constituye como un impuesto al consumo que incide en la producción nacional y extranjera que a su vez otorga reembolso a la exportación. El tipo de bienes sujetos al IEPs en nuestro país no es muy diferente al de otros países miembros de la ocDE, sin embargo sus características específicas sí lo son.

Algo en común que tienen los impuestos especiales es que gravan bienes y servicios específicos que observan una curva de demanda inelástica o bien con poca elasticidad, por lo tanto son una alternativa para fortalecer los ingresos de un país sin incurrir en grandes distorsiones económicas por lo menos en el corto plazo. No obstante, es probable que un sistema tributario ineficiente pueda propiciar menores ingresos en la medida que una mala determinación del gravamen pueda originar la aparición de mercados negros, o bien, el contrabando de dichos productos. ${ }^{10}$

Hablando de cantidades recolectadas, como se comentó arriba, en promedio durante el periodo de 1977 a 2003 este impuesto participaba con un 17\% del total de la tributación del país, aun cuando a través del tiempo ha mostrado variaciones fuertes con valores que van de $9.7 \%$ como mínimo a 24.4\% como máximo en la recaudación (véase gráfica 6, p. 124).

Para tratar de explicar la variación de la capacidad de ofrecer recursos al fisco por parte de este impuesto hay que desglosar las partes que lo integran. Este impuesto se integra o constituye por: combustibles, cerveza, vinos, bebidas alcohólicas destiladas y tabaco. ${ }^{11}$ No obstante que el impuesto está integrado por varios bienes, son aquéllos que gravan los bienes provenientes del petróleo los que representan la mayor parte de los ingresos recaudados por el IEPS (en promedio para el periodo aproximadamente el $63 \%)$; por lo tanto, no es nada ilógico pensar que son las medidas relacionadas a la gasolina y el diesel lo que estará determinando el comportamiento y variación de la

\footnotetext{
${ }^{10}$ Trigueros y Fernández (2001) incluso mencionan que bajo ciertas circunstancias la elevación del gravamen en estos bienes y servicios puede no ser una alternativa atractiva para fortalecer la recaudación, ya que se pueden ocasionar incentivos para el contrabando y otras actividades informales.

${ }^{11}$ En el Artículo $2^{\circ}$ de la "Ley del Impuesto Especial sobre Producción y Servicios”, publicada en el Diario Oficial de la Federación el 30 de diciembre de 1980 y observando su última reforma publicada en el Diario Oficial de la Federación el $1^{\circ}$ de diciembre de 2004, se enumeran 7 tipos de bienes y un servicio, que son susceptibles de la aplicación del impuesto; los bienes son: a) Bebidas con contenido alcohólico y cervezas, b) Alcohol, alcohol desnaturalizado y mieles incristalizables, c) Tabacos labrados, d) Gasolinas, e) Diesel, g) Refresco, y g) Jarabes o concentrados para preparar refrescos. En el caso del servicio, se tiene que: la comisión, mediación, agencia, representación, correduría, consignación y distribución, con motivo de la enajenación de los bienes señalados en los incisos a), b), c), g) y h), serán también objeto de este impuesto.
} 
recaudación derivada del IEPS y, en este sentido, el impuesto se desvincula del comportamiento de la producción agregada como se comentaba en paginas anteriores.

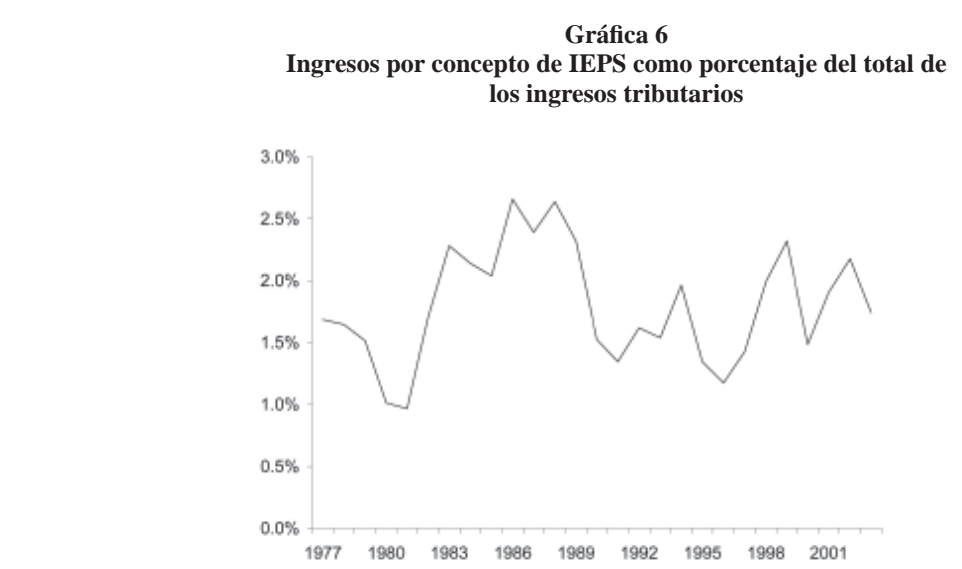

Fuente: Elaboración propia con datos del Sistema de Finanzas y Deuda Pública, Subsecretaría de Hacienda y Crédito Público y Dirección General de Planeación Hacendaria.

Con respecto a los bienes no petrolíferos los más importantes, listados en ese orden, son los tabacos labrados, la cerveza y el alcohol. Una ventaja adicional del IEPS, de conformidad con Revilla y Zamudio (2001), es que el impuesto resulta en términos absolutos progresivo; no obstante que cuando se estudia de manera separada la incidencia para el consumo de bebidas embriagantes y tabaco el resultado es una afectación mayor a los hogares más pobres, y en este sentido es parcialmente regresivo. Pero, dado que para los productos petrolíferos es progresivo y estos constituyen más del $60 \%$ del impuesto, el resultado es un impuesto progresivo.

Después de haber ofrecido algunos datos de los tres principales impuestos en el país, así como algunas de sus limitaciones para conseguir una mayor recaudación, se revisarán las características mínimas que debería presentar una estructura tributaria para considerarse eficiente.

\section{Estructura tributaria}

La estructura tributaria es un sistema financiero que limita o contrae el gasto privado permitiendo con ello el gasto público; la manera en que este sistema financiero extrae los recursos de los agentes privados es mediante el establecimiento de diversos impuestos que pueden gravar en términos generales la renta o el consumo. 
Crítica a la estructura tributaria actual en México

El problema de un sistema financiero como éste consiste en que no sólo extrae recursos de los individuos de una sociedad, sino que también puede tener un efecto importante al modificar la conducta de los agentes económicos. En este sentido se considera la existencia de dos tipos de impuestos, los denominados distorsionadores y aquéllos que no lo son.

Un impuesto es no distorsionador si, y sólo si, los agentes económicos no pueden hacer nada para alterar sus obligaciones fiscales; este tipo de impuestos en la literatura fiscal es conocido como impuestos de suma fija o bien de capitación. Los impuestos de suma fija son aquéllos que no dependen de la renta o la riqueza o del consumo y, por lo tanto, no pueden distorsionar la conducta de los individuos.

La estructura tributaria en la gran mayoría de los casos se encuentra conformada por impuestos que distorsionan la economía, por lo tanto ninguna de ellas puede favorecer el crecimiento económico en un sentido absoluto; parece claro que si no hubiera impuestos al consumo las empresas venderían más y los individuos adquirirían más satisfactores. Asimismo, si las empresas no vieran gravada su renta podrían favorecer más la creación de empleos y el desarrollo económico.

Desde luego que es necesario entender que las acciones del Estado no pueden ser gratis y por lo tanto la discusión debería centrarse en la posibilidad de conformar una estructura tributaria que recaudando la misma suma de ingresos pueda tener un perjuicio menor para el crecimiento económico y no sólo eso, sino avanzar, incluso, en la consolidación de una estructura tributaria bien diseñada que participe en un crecimiento de largo plazo.

En una conferencia que tuvo lugar en el año 2003 Jean-Philippe Cotis, economista en jefe de la OCDE, mencionaba que en una economía con gasto óptimo limitado por el nivel de ingresos tributarios se podría decidir entre tres alternativas: Incrementar la deuda pública, aumentar la recaudación de impuestos o mantener niveles de gasto bajo y volátiles. En su opinión la segunda opción era la más adecuada, por lo tanto se tendría que proceder a incrementar la recaudación para financiar los gastos que tienen altos beneficios económicos y sociales y, al mismo tiempo, mantener un gobierno pequeño y minimizar la carga tributaria mediante una estructura fiscal óptima.

Para lograr que una estructura tributaria pueda recaudar la misma cantidad de recursos afectando menos al crecimiento económico, es necesario hacerla más eficiente. De acuerdo con Stiglitz (2002: 483-508), para que una estructura tributaria sea efi- 
ciente se tendrían que observar cinco atributos fundamentales: Eficiencia económica, sencillez administrativa, flexibilidad, responsabilidad política y justicia. ${ }^{12}$

\section{Eficiencia económica}

Se dice que una estructura tributaria no debe de interferir en la asignación eficiente de los recursos; por el contrario, debería de utilizarse para aumentar la eficiencia económica. Dado que cualquier sistema tributario influye en la conducta de las personas, el objetivo será buscar la combinación de instrumentos (impuestos) que ofrezcan la menor respuesta de los agentes económicos y por lo tanto se obtenga una menor distorsión.

Al parecer existe coincidencia entre varios autores al respecto de que los impuestos enfocados al consumo distorsionan menos que los que usan como base la renta (Doménech y García, 2000). Si es correcta esta apreciación muchos se inclinarían porque la estructura tributaria estuviera conformada proporcionalmente por un mayor número de impuestos dedicados al consumo en lugar de la renta, evitando con ello crear distorsiones en la correcta asignación de los factores y dejando así que el mercado realizara la mejor asignación posible de acuerdo con Pareto.

Para el caso de México, y tomando en cuenta las cifras vertidas con anterioridad, se puede argumentar que el impuesto al consumo y el dedicado a la renta recaudan la misma proporción; por lo tanto, en el afán de no distorsionar la economía se tendría que avanzar en modificar la estructura hacia una relación de mayor peso para los impuestos al consumo (impuestos indirectos). Sin embargo, en la búsqueda de la eficiencia económica se podría afectar al menos otro de los atributos de la estructura fiscal y ésta es la justicia. ${ }^{13}$

Si se saca la discusión del lado de los ingresos, y se pasa al de los gastos, surge otro problema que consiste en determinar la equidad del gasto público o su incidencia;

\footnotetext{
${ }^{12}$ Hernández et al. (2000) menciona que, aun cuando no hay consenso sobre las características específicas de un sistema tributario, existen lineamientos generales que favorecen su buen funcionamiento como son: interferir lo menos posible en la eficiente asignación de los recursos; tener una administración sencilla y relativamente barata; ser flexible para responder a los cambios y circunstancias económicas, políticas y sociales; cumplir con los principios de equidad y proporcionalidad, y mostrar transparencia, a fin de que los individuos sepan hacia donde se dirigen sus contribuciones.

${ }^{13}$ Musgrave (1995: 13) comenta que aún cuando la redistribución implica inevitablemente un costo de eficiencia, esta situación no debería ser motivo para dejarla de lado, pero se deberá tener cuidado en que cualquier cambio distributivo se logre con el menor costo de eficiencia posible ya que existe la necesidad de los objetivos de eficiencia y equidad, cualquier política óptimamente dirigida deberá considerar estos dos elementos.
} 
Crítica a la estructura tributaria actual en México

con lo que de todos modos se tendría que observar justicia en la asignación de los recursos provenientes de los ingresos tributarios y se quedaría donde se empezó. La asignación del gasto en México de acuerdo con Scott (2001) es regresivo en términos absolutos, es decir, que en el saldo total la asignación del gasto beneficia más a los de mayores ingresos; si a esto se le asocia una estructura tributaria regresiva, será complicado cumplir con la quinta característica de una estructura tributaria eficiente.

Otro elemento que debe ser ampliamente valorado en este punto consiste en los efectos que los impuestos regresivos pueden tener sobre la demanda agregada. Si los impuestos son regresivos, como ya se había comentado, gravan más a las personas de menor ingreso y en este sentido, siguiendo el análisis keynesiano, se puede disminuir la demanda agregada al disminuirse el consumo. Lo anterior partiendo de la idea de que el consumo de los agentes privados es diferente de acuerdo con su ingreso. ${ }^{14}$ Los impuestos al consumo al disminuir la demanda agregada inhiben la inversión e impiden el crecimiento económico. Si se da una disminución del crecimiento económico y dada la prociclidad de los impuestos, también se disminuirá la captación tributaria.

\section{Sencillez Administrativa}

Cualquier estructura tributaria debería de presentar un diseño de tributos que sean sencillos, fáciles y relativamente baratos en su administración por parte de las autoridades y su cumplimiento por parte de los ciudadanos.

Cualquier estructura tributaria tiene al menos dos tipos de costos administrativos: los directos e indirectos. En el caso de los primeros son los que devienen de gestionar la oficina de recaudación, mientras que los segundos son aquéllos que deben ser pagados por los contribuyentes en el cumplimiento de su obligación fiscal.

Entre más compleja resulte ser la estructura tributaria se incurrirá más en cada uno de los costos incrementando con ello la carga fiscal de los contribuyentes que no encuentran una contraprestación y que, por lo tanto, distorsionan en mayor medida las decisiones de los agentes económicos. La carencia de sencillez administrativa en la composición de la estructura fiscal puede determinar o incidir en el grado de evasión fiscal. No obstante, la complejidad de la estructura tributaria se origina por la búsqueda de una estructura tributaria progresiva.

\footnotetext{
${ }^{14}$ Las personas de menores ingresos tienen una propensión a consumir más alta. Peacock y Shaw (1974: 32-33), mencionan que los “trabajadores” tienen una propensión marginal a consumir igual a la unidad y los “capitalistas” una propensión menor a la unidad.
} 
Eduardo Ramírez Cedillo

\section{Flexibilidad}

Una estructura tributaria debería de ser capaz de responder fácilmente a los cambios de las circunstancias económicas, cuando la economía se encuentre en expansión los ingresos tributarios se deberán incrementar, mientras que cuando la economía está en recesión o en una fase descendente los ingresos tributarios también lo hacen, por lo tanto son pro-cíclicos.

Si los ingresos tienen el mismo comportamiento del ciclo económico sería difícil sustentar una reactivación de la economía mediante la misma postura fiscal, por lo que sería recomendable actuar con una política fiscal contra-cíclica. La manera como funciona una política fiscal contra-cíclica es disminuyendo la carga fiscal en los momentos de recesión e incrementándolos cuando se presenten choques de demanda que puedan traer consigo desequilibrios en el nivel de precios.

Sin embargo, para que la estructura tributaria observe flexibilidad se enfrenta a dos contratiempos: las dificultades políticas y la velocidad de ajuste. Por ambos motivos Arestis y Sawyer (2003a) mencionan que se ha privilegiado el uso de la política monetaria para situaciones de estabilización, ya que sus decisiones no pasan por un proceso legislativo. No obstante, se argumenta que si la política fiscal actúa de manera inmediata porque tiene objetivos de inflación el objetivo de la política fiscal podría ser una tasa de desempleo, con lo que se agilizarían los ajustes mediante la política fiscal evitando las complejidades legales y que las medidas correctivas pudieran tener rezagos en su aplicación.

México es un buen ejemplo de lo complejo que puede resultar contar con una estructura tributaria flexible. La reforma en materia tributaria, propuesta por el Ejecutivo, no ha podido ser aprobada; pero no sólo eso: independientemente de los juicios de valor al respecto, la actitud de los congresistas parece conducirse bajo el principio de "que ante la duda la inactividad". Si bien la propuesta del Ejecutivo no es la correcta deberíamos tener otras propuestas alternativas y haber implementado alguna política que sea coherente con la situación económica del país. La flexibilidad en un momento dado no implica que todas las decisiones sean las idóneas, pero sí garantiza que de no ser así puedan ser mejoradas de forma relativamente oportuna.

\section{Responsabilidad política}

Se dice que una estructura tributaria observa responsabilidad política cuando los ciudadanos tienen claro quiénes se benefician y quiénes tienen que pagar. En este sentido el impuesto sobre la renta de las personas físicas es un impuesto altamente transpa- 
Crítica a la estructura tributaria actual en México

rente, donde el contribuyente por lo menos sabe con cuánto participa su ingreso en la recaudación, mientras que el impuesto al consumo puede ser poco transparente ya que los individuos normalmente nunca calculan la cantidad total que pagan por concepto de consumo y peor aún si se enfrenta a diferentes impuestos que gravan actos de consumo, como los impuestos especiales, a la importación, por la compra de autos nuevos, etcétera.

La ventaja de un impuesto sobre el ingreso de las personas físicas contra el impuesto al consumo consiste en que el primero es descontado en una sola exhibición del ingreso que percibe el contribuyente, por lo tanto él puede notar de manera inmediata la afectación que tiene; mientras que en el segundo la afectación es gradual con cada acción de consumo. En la práctica cualquiera de las dos opciones tiene la misma afectación sobre el contribuyente, disminuye su capacidad de consumo, pero en su percepción puede ser que estime una afectación mayor en el dirigido a la renta que en el consumo.

A este respecto de la transparencia, el gobierno de Vicente Fox ha dado un paso de gran trascendencia al establecer la "Ley de Transparencia y Acceso a la Información Pública Gubernamental”, publicada en el Diario Oficial de la Federación el 11 de junio de 2002. Este ordenamiento se cristalizó en materia tributaria en el Decreto por el que se reforman, adicionan y derogan diversas disposiciones contenidas en la Ley del Servicio de Administración Tributaria, publicado en el Diario Oficial de la Federación el 12 de junio de 2003. En dicho decreto se adicionó un Título Quinto denominado "De la Información, la Transparencia y la Evaluación de la Eficiencia Recaudatoria y de Fiscalización”, donde se hace énfasis en poner al alcance de la sociedad información de diversa índole como puede ser: Las medidas realizadas para eliminar la corrupción, la cantidad recaudada por tipo de impuesto, los juicios ganados por el fisco federal en materia de tributación, indicadores de eficiencia, etcétera.

\section{Justicia}

Al hablar de justicia como una característica que debe presentar una estructura tributaria eficiente se hace mención a la equidad; solamente es justo lo que es equitativo, por lo tanto lo justo sería que de manera equitativa los agentes económicos participaran con sus obligaciones fiscales.

En la teoría tributaria se manejan dos tipos de equidad, la equidad horizontal y la vertical. En la primera se reconoce que individuos que son iguales en los aspectos fundamentales deben recibir un mismo trato; no obstante, la segunda menciona que algunos individuos se encuentran en mejores condiciones que otros para pagar im- 
puesto y que por lo tanto deben hacerlo. Sin la intención de profundizar en aspectos filosóficos al respecto de la igualdad y la justicia se avanzará en la idea bajo la cual la estructura tributaria puede obrar con equidad.

La equidad vertical supondría que los impuestos que pagan los individuos con mayores ingresos son mayores a los que pagan los individuos con menores ingresos. Los impuestos que logran la equidad vertical son aquéllos que son denominados progresivos ya que recaudan un porcentaje mayor del ingreso cuanto mayor sea éste; mientras que los regresivos recaudan un porcentaje mayor cuanto menor es el ingreso.

Si se desea mantener la equidad del sistema se tendrá que incurrir en dos situaciones por lo menos: la distorsión económica negativa que origina un impuesto progresivo y los costos administrativos asociados a la complejidad relacionada a los impuestos progresivos. Ambas situaciones podrían ser corregidas mediante la utilización de los impuestos regresivos a cambio de incumplir con la equidad.

Después de revisar las cinco características básicas para lograr una estructura tributaria eficiente, parecería que se está en una disyuntiva en la cual se pueden conseguir unos puntos en detrimento de otros; no obstante, si se abre la visión y se deja de ver la estructura tributaria como un fin en sí misma, para verla como un medio, se puede pasar a otro orden de ideas. Si la estructura tributaria forma parte de las decisiones de política fiscal, y ésta persigue el crecimiento económico sostenible en el largo plazo, se tendría que analizar el efecto de los impuestos en el logro del crecimiento económico de largo plazo, sin embargo esta labor es más profunda que los objetivos de este ensayo.

A continuación se describe la propuesta de reforma tributaria realizada por el Ejecutivo Federal y algunas otras alternativas.

\section{La propuesta de reforma realizada por el Ejecutivo y otras alternativas}

El 3 de abril del 2001 el Poder Ejecutivo envío a la Cámara de Diputados una iniciativa fiscal y financiera denominada "La Nueva Hacienda Pública Tributaria”, dicha propuesta en grandes rasgos consistían en:

> La generalización del 15\% del IVA a los alimentos y medicinas.

$>$ Disminución del ISR haciéndolo más fácil de calcular. ${ }^{15}$

$>$ Eliminación de los regímenes especiales.

${ }^{15}$ Revilla y Zamudio (2001) presentan un cuadro resumen de las modificaciones que se propusieron por parte del Ejecutivo. 
Crítica a la estructura tributaria actual en México

La reforma original consistía básicamente en un movimiento de tasas entre el Impuesto Sobre la Renta (ISR) y el Impuesto al Valor Agregado (IVA), por medio del cual se pretendía disminuir la carga fiscal del ingreso y elevar el impuesto recaudatorio en el consumo.

La propuesta del Ejecutivo resulta consistente con la visión del Banco Mundial (Reyes, 2001) y de la OCDE (Cotis, 2003), quienes estiman que los recursos adicionales deberán venir del impuesto al IVA, mediante la eliminación de los regímenes de exención y la generalización de la tasa. La razón de enfocarse en el IVA consiste en el hecho de considerarlo como un impuesto que genera distorsiones mínimas y al mismo tiempo recauda los ingresos de una base relativamente estable.

En la literatura tributaria no hay elementos concluyentes al respecto de las modificaciones que se pretenden realizar al IVA. Velarca (2002) presenta evidencia a favor de los impuestos al consumo para los años 1994 y 2000, donde encuentra que no sólo no se empeoró la distribución del ingreso, sino que incluso se mejoró; aun cuando él mismo reconoce que el periodo de estudio es corto, agrega que de comprobarse su estudio para otros años los impuestos al consumo podría ser una herramienta importante para fomentar la equidad en el ingreso sin causar distorsiones. Por su parte, Revilla y Zamudio (2001) usando la ENIGH-98 encuentran que la propuesta presidencial hace regresivo el impuesto. En otro orden de ideas se tiene una visión interesante propuesta por Marcelo (2001), quien menciona que antes de realizar cambios en los tipos impositivos se debería de empezar por modificar y poner en orden la estructura administrativa que maneja, audita, supervisa, evalúa y administra la estructura tributaria, ya que de no ser así los resultados no serán los deseados.

\section{Conclusiones}

A manera de conclusión se destacan algunas ideas principales:

- En primer lugar resulta evidente que la capacidad de recaudación de la estructura tributaria actual tiene deficiencias que impiden cerrar la brecha entre los ingresos potenciales y los observados.

- Los nuevos estudios al respecto de la evasión fiscal apuntan a la simplificación de los impuestos, lo que implicaría abandonar los regímenes especiales y las exenciones.

- Resulta claro que las propuestas en la modificación de la composición de la estructura tributaria deben de ir acompañadas de la aplicación de gasto en programas sociales que tenga por objeto tratar de revertir los efectos regresivos que presentan los impuestos al consumo.

- Una reforma a la estructura fiscal debe ser integral; no se puede limitar tan sólo a la modificación de las tasas impositivas. Se debe distinguir qué parte del impuesto se 
pierde por las características del mismo y cuáles están asociadas a la institución que se encarga de administrarlo.

Un comentario final, que quizás no estuvo muy presente a lo largo del escrito, es el siguiente. La mejor manera de incrementar la recaudación de un país es la reactivación económica, pues mediante ella se generan más trabajos formales, se amplia la rentabilidad de negocios permitiendo que los informales puedan tener si no el deseo voluntario, sí la capacidad de cumplir con sus obligaciones fiscales. Desgraciadamente mientras las políticas tributarias se mantengan más que pro-cíclicas, y exista el ánimo de mantener la disciplina fiscal, las posibilidades de crecimiento son insuficientes.

\section{Bibliografía}

Arestis, P. y M. Sawyer (2003a). Reinventing Fiscal Poucy, Working Paper, mayo, núm. 381. Nueva York: The Levy Economics Institute of Bard College.

(2003b). The Case for Fiscal Policy, Working Paper, mayo, 382. Nueva York: The Levy Economics Institute of Bard College, mayo de 2003.

Ayal a, J. (2001). Economía del Sector Público Mexicano (2ª ed.). México: Esfinge.

Cantalá, D. et al. Evasión Fiscal en el Impuesto sobre la Renta de Personas Físicas. El colegio de México. Extraído en enero de 2005 desde ftp://ftp2.sat.gob.mx/asistencia_servicio_ftp/publicaciones/estudios_ef/EvFiIsrPFAr06.pdf

Cot is, J. (2003). Opinión de la OCDE sobre la Reforma Tributaria en México. Conferencia llevada a cabo los días 14 y 15 de octubre en la ciudad de México

Decreto por el que se reforman, adicionan y derogan diversas disposiciones contenidas en la Ley del Servicio de Administración Tributaria, DOF, México, 12 de junio de 2003.

Díaz E. y J. Mendoza (2005). Evasión Fiscal en el Impuesto Sobre la Renta Retención de Salarios, El Colegio de la Frontera Norte. Extraído en enero de 2005 desde http://www.sat.gob.mx/sitio_internet/transparencia/51_3321.html

Doménech, R. y J. Gar cía (2000). Estructura Fiscal y Crecimiento Económico en la OCDE. Extraído en julio de 2000 desde http://iei.uv.es/ rdomenec/efce_1.html

East er ly, W. y S. Rebel o (1993). Fiscal Policy and Economic Growth: An Empirical Investigation, Working Paper, 4499, octubre 1993. Massachusetts: NBER. 
Crítica a la estructura tributaria actual en México

Engen E. y J. Skinner (1996). TaXation and Econom ic Growth, National Tax Journal (pp. 617-642), vol. 49, 4, diciembre 1994.

Gemmel l, N. (2001). Fiscal Policy in a Growth Framework, Discussion Paper, núm. 2001/48, septiembre 2001. Helsinki: UNU/Wider, World Institute for Development Economics Research.

Gil, F. y W. Thirsk (2000). La prolongada Reforma Fiscal en México, Gaceta de Economía, Suplemento, año 5, núm. 9. México: ITAM.

Her nández, F. et al. (2000). Los Impuestos en México: ¿Quién los paga y Cómo? En Programa de Presupuesto y Gasto Público. México: Centro de Investigación y Docencia Económicas.

Her nández, F. y A. Zamudio (2004). Evasión Fiscal en México: el Caso del IVA. Extraído en enero 2004 desde ftp://ftp2.sat.gob.mx/asistencia_servicio_ftp/publicaciones/estudios_ef/eef_iva.pdf

Ibarra, D. (2001). Las Tributaciones Hacendarias, en Economía Informa (pp. 60-67), núm. 303, noviembre de 2001.

Ley de Transparencia y Acceso a la Información Pública Gubernamental, 11 de abril de 2004. México: DOF.

Ley del Impuesto al Valor Agregado, 01 de diciembre de 2004. México: DOF.

Ley del Impuesto Especial sobre Producción y Servicios, 01 de diciembre de 2004. México: DOF.

Musgr ave, R. y P. Musgr ave (1995). Hacienda Pública, Teórica y Aplicada (5ª edición). México: McGraw Hill.

Myl es, G. (2000). Taxation and Economic Growth, Fiscal Studies (pp.141-168), vol. 21, núm. 1.

Peacock, A. y G. Shaw (1974). La teoría Económica de la Política Fiscal. México: FCE.

Revil la, E. y A. Zamudio (2001). Reforma Fiscal y Distribución de la Carga Impositiva en México, Programa de Presupuesto y Gasto Público. México: Centro de Investigación y Docencia Económicas.

Reyes M., Problemas Estructurales del Sistema Impositivo y Propuesta de Reforma Tributaria en México, Cámara De Diputados, Servicio de Investigación y Análisis, División de Economía y Comercio México, 2001. 
Romer, P. (1990). Endogenous Technological Change, The Journal of Political Economy (pp. 71-102), vol. 98, núm. 5, octubre 1990.

Rosen, H. (2002). Hacienda Pública (5ª ed.). España: McGraw-Hill.

Scout, J. (2001). La otra cara de la reforma Fiscal: la Equidad del gasto público, Programa de Presupuesto y Gasto Público. México: Centro de Investigación y docencia económicas.

St igl it z, J. (2002). La economía del Sector Público (3ª ed). España: Antoni Bosch.

Triguer os, I. y A. Fer nández (2001). Análisis Evaluación y Propuesta para una Reforma Tributaria, Una Agenda para las Finanzas Públicas en México. México: ITAM.

Vel arca, A. (2002). Los Impuestos en México y sus Efectos en la Distribución del Ingreso, Gaceta de Economía, año 8, núm. 15. México: ITAM.

Zagl er, M. y G. Dür necker (2003). Fiscal Policy and Economic Growth, en Journal of Economic Survey (397-418), vol. 17, núm. 3. 